\title{
Design and implementation of grid based clustering in WSN using dynamic sink node
}

\author{
Aya Ahkam Kamil ${ }^{1}$, Maham Kamil Naji ${ }^{2}$, Hasan Abdulhadi Turki ${ }^{3}$ \\ ${ }^{1}$ Middle Technical University, Electrical Engineering Technical College, Iraq \\ ${ }^{2}$ Middle Technical University, Institute of Technology-Baghdad, Iraq \\ ${ }^{3}$ Ministry of Construction and Housing, Iraq
}

\begin{tabular}{l} 
Article Info \\
\hline Article history: \\
Received Oct 25, 2019 \\
Revised Feb 6, 2020 \\
Accepted Apr 29, 2020 \\
\hline
\end{tabular}

\section{Keywords:}

Grid based clustering

Sink node position

Static \& dynamic

WSN

\begin{abstract}
A wireless sensor networks (WSNs) play a significant application, especially in the monitored remoting environmental, which enables by the availability of sensors which are cheaper, smaller, and intelligent. The equipment of such sensors be with wireless interfaces, which a communication with other sensors occurs for creating a network, that contains many distributed nodes. The closest nodes to the sink are exploited at an enormous traffic load while the data from the whole regions are forwarded between them to reach the sink. This result in exhausting their energy quickly and partitioning the network. This is solved by changing the sink node position in Grid based clustering technique, which considers the optimal method for this purpose. A simulation with MATLAB can be applied for grid based clustering technique to evaluate the performance of WSN. The expected results deal with outperforms in throughput, reducing energy consumption and increasing residual energy, in addition to prolong the network lifetime of the sensor network.
\end{abstract}

This is an open access article under the $\underline{C C B Y-S A}$ license.

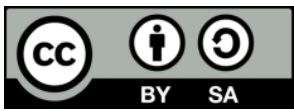

\section{Corresponding Author:}

Aya Ahkam Kamil,

Electrical Engineering Technical College, Departement of Control and Automation

Engineering Techniques,

Middle Technical University,Baghad, Iraq.

Email: dradnanmtu@gmail.com

\section{INTRODUCTION}

A currently, a wireless sensor networks (WSNs) are among the most essential technologies, which consists of huge number of small nodes having a communication, computation in addition to sensing capabilities. Such sensor nodes are confined with energy, battery, transmission range, memory, ability of processing, and distributed over a large area [1]. The data losing results from the damage in undesired physical and environmental, power or blockage of any obstacle form are not available.

WSN is beneficial for monitoring numerous applications, like surveillance, security, disaster management, military, healthcare and environmental studies, so that, WSNs have become the most tremendous area of research [2]. The rate of energy consumption and the WSN transmission overhead may be decreased during the aggregated data generation, and the forwarding of the data aggregation. The division of nodes into small groups is identified as clusters, that enable the data aggregation inside the network. The clustering process refer to the nodes dividing into groups constructed on certain mechanism. An employing of clustering is to improve lifetime of the network, that is considered the major parameter for assessing the sensor network performance [3]. 
The energy of the nodes can be considered very important criteria to prolong the lifetime and to increase the performance of wireless sensor network. So, the nodes energy near the sink is exhausted very fast in hierarchical protocols where the sink node in fixed position, which leads a network get separated. A sink node can be used under dynamic position for improving energy balancing entire network to overcome this problem and to extend the network lifetime [4]. As a result, the collecting data from the sensors to the sink node can be transferred along with fewer power consumption and more efficient routing that avoiding the delay in relaying events [5].

Many discussions were presented in previous works that had been approved with the purposing of addressing issues associated to the prolonged lifespan of the network, while balancing the energy consumption in wireless sensor networks Amrutha \& Ashwini (2013) [6] described WSN as a group of small, low-cost and self-powered devices that capable of sensing, computing and communicating with other devices with a view of collecting limited information that can enable global decision-making about the physical environment.

The performances of the most three routing protocols in WSN grid based clustering were compared by Pratibha \& Laxmi (2014) [7]. The sensor nodes limited energy and battery power were maintained by energy sensor networks; the major task of the sensor network, is the energy consumption of sensor nodes. In addition, the functions of the grid-based sensor were based on the locations that are divided into many parts, while the cluster head in grid based WSN, considered as a base station. The three routing protocols were; Dynamic Manet on demand routing (DYMO), Ad-hoc on-demand distance vector routing (AODV) and Dynamic source routing (DSR) through the use of Qualnet simulator.

Cunxiang \& Zunwen (2014) [8] proposed a LEACH protocol to decrease the energy cost, due to the insufficient energy of WSNs. The routing role for improving energy efficiency can't be underestimated. LEACH problem concentrated on no attention paid to the residual energy of sensor nodes and long-distance communication, thus high energy consumption resulting and low network coverage. They proposed a grid-based energy efficient routing protocol (GEERP) that focused to improve these three things (energy consumption, network coverage and energy efficiency).

According to Bhaskar, et al. (2016) [9], major factors were considered in the WSN design which are the energy conservation and load balancing. The methodology of clustering assists in energy reducing that is consumed by the network nodes, thereby results in an increasing in the network lifespan.

Shrijana \& Kalpana (2016) [10], directed to design an energy efficient cluster head selection and rotation mechanism, which leads to a whole communication can be occurred between the base station and the cluster that take care of by the cluster head. In a cluster, originally, the cluster members are consuming less energy than a cluster head, because it receives data from all members, senses the data, processes the data and communicates the process result in the base station.

According to Ashwini \& Asha (2017) [11], they explained that the consumption of energy is the key issue in the WSN design. They used a clustering method which considered a way through which the energy consumption by each node can be reduced, so that the lifetime of the network can be prolonged. The paper also aimed to address the energy loss problem which occurs in the clusters that are located close to the BS as a result of more data handling.

Based on a grid structure in WSNs, a new algorithm for routing and clustering was proposed by Lalitha et al. (2017) [12]. They calculated an appropriate grid size and constructed a virtual grid structure, based on the size of the area and range of transmission. The selection of a cluster head within a grid is done based on closest distance to the midpoint of the grid. Bilal et al (2017) [13], noted that minimizes energy consumption has been achieved by clustering hierarchical schemes. In their paper, the hierarchical schemes were broadly classified as cluster-based and grid-based approaches. Cluster-based approaches involve the grouping of nodes into clusters, with one relevant sensor node selected as the cluster head, while grid-based approach involves the division of the network by the base station into confined virtual grids. Najma, et al. (2018) [14] demonstrated a dependable data communication with maximum lifetime of the network are essential by WSN applications, that are more fundamental in Mobile Wireless Sensor Networks (MWSN) compared with static WSN. This is due to the reliability of receiving data at the cluster head in cluster based MWSN is very challenging. Zhansheng \& Hong (2018) [15], proposed a reliable grid-based multi-hop routing approach for WSNs, with the aim of minimizing and balancing the consumption of energy. The proposed protocol has the capability of optimizing the process of cluster head selection through a combination of individual ability.

This paper is focused on proposing a grid-based clustering design under dynamic sink node position for balancing the traffic load in the network and, to implement a design of the grid-based clustering in multiple cases and compare between them for identifying appropriate network design in WSN by using MATLAB. 


\section{RESEARCH METHOD AND MATERIALS}

\subsection{Grid based in WSN}

In WSN model, the grid shape is squared, and shape of each square is a zone made. The rows and columns numbers are symbolized by $n \times n$. A rows and columns grouping represents the grid, the left to right and top to bottom. Rows starting from the left side to the right (n), whereas the columns are top start and bottom end (n). The network model of $2 \times 2$ grid-based is explained in Figure 1(a), Ri represents the rows, whereas $\mathrm{Cj}$ is columns represent [16]. Figure 1(b) demonstrates the field of sensor network, where the grids have been divided. The zone of the network contains square grids with a non-overlapping and equal sizes and every grid principally must contain a master working node every time. Consequently, the nodes of the grid must have a sequence task one after another, which leads to the network lifetime to be prolonged $[17,18]$.

A principle working of any grid expects having one node, considers the head, which has the forward responsibility of the routing information and data packets transmission. The execution of the Routing is completed grid-by- grid way. The purposing of the multi-path routing protocol in grid-based clustering is a packets rapid routing ability, exploitation and extend the sensor nodes energy, while avoiding the network congestion, or treating it, if it happens $[19,20]$

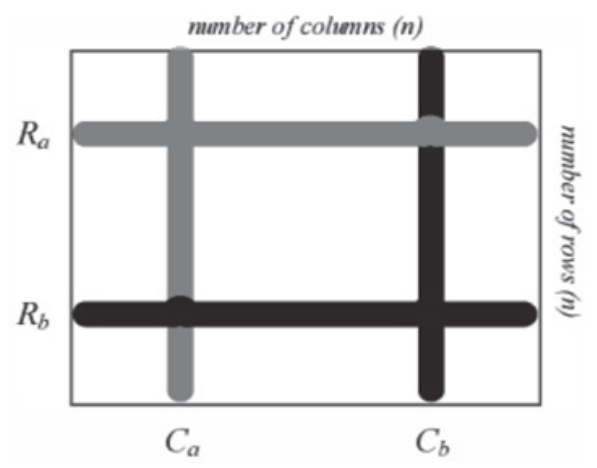

(a)

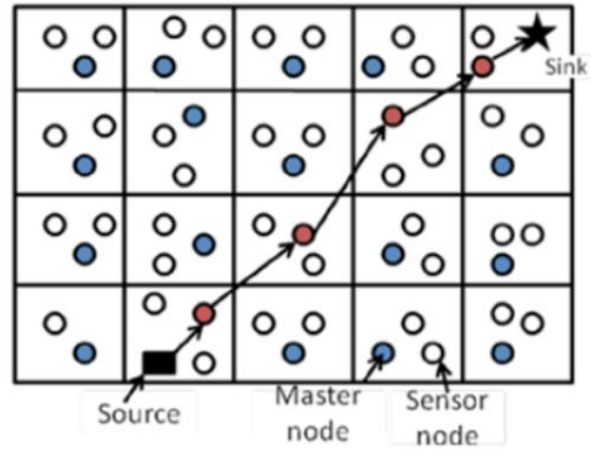

(b)

Figure. 1 Grid topology, (a) $\mathrm{n} \times \mathrm{n}$ grid, (b) A wireless sensor network grid

The purpose of a WSN grid-based clustering technique can be concerned for enhancing the minimization of the node power consumption by minimizing the traffic load. A usefulness of grid based clustering head $(\mathrm{GBCH})$, comprise the sensing field partitioning through grids with equal square size, the $\mathrm{CH}$ selection at any grids, increasingly the network lifespan, and improving the energy efficiency [21]. This technique contains two important phases, the first is initial clustering and the second is data transmission. Many items have an initial clustering such as the grid construction, the selection of $\mathrm{CHs}$, in addition to the scheduling of TDMA, whereas the focusing of data transmission phase on the packets forwarding to the destination from the $\mathrm{CHs}$ [22].

On the other hand, the high rate problem of the energy consumption remains a matter of great attention in WSNs. So that, a reducing of the energy consumption is essential to enhance the performances of prolonging network lifespan and to decrease the sensor node congestions that is considered the actual responsibility to balance the load of the network. At the sink, a larger number of packets will be received in different topologies like mesh, circular and grid mostly.

\subsection{Sink node position in WSN}

WSN with sink node architecture can be distributed with static and dynamic positions, it consists of sensor nodes, or mobile sink nodes, which sometimes it can be used as a data collector that is installed within the sensing area. The static sink node position could be utilized for WSN collecting data by forwarding multi hop which gives an advantage of consumption more energy nearby nodes around Base station in addition to relay the data from another one.

The second is the dynamic sink node position that is also could be used for collecting data from sensor nodes and storing it at the base station through a single round in the network, thus with a new round the sink node consumes a new position to balance the energy consumption within multiple nodes full network. 


\subsection{Research methodology}

The proposed techniques for the improvement of WSN performance are demonstrated in details with a suggested algorithm for grid-based cluster. Two proposed scenarios for Simulation design are realized using a set of nodes. The construction of these scenarios is carried out by using MATLAB, which is currently top used simulation design. Many parameters are used to evaluate the WSN performances with grid-based clustering techniques including essentially consumption of energy during transmission, throughput, in addition to network lifetime.

The proposed flow chart consists of two phases that will be implemented with two scenarios static and dynamic sink node position and then a comparison will be made to reach an appropriate network design Building as shown in Figure 2. These two phases are construction and connection, in construction, the design of two scenarios in different algorithm are built, while a connection Phase is responsible for creating a connection between nodes.

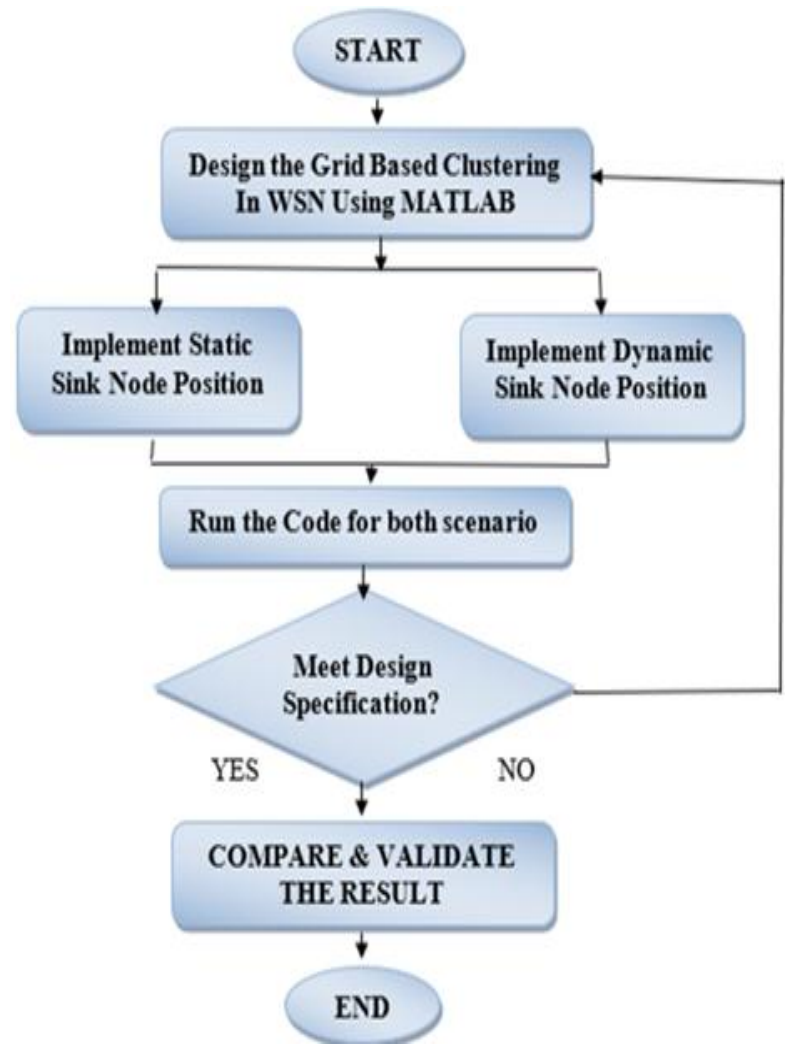

Figure 2. Overall methodology

\subsection{Simulation scenarios}

A simulation of two scenarios has been done; the first is the grid-based clustering design under static sink node position while the second is for dynamic. Each scenario consists of 800 sensor nodes in the simulated design, these nodes distributed randomly on number of grids is $4 * 4$ at a surface area of 800 by $800 \mathrm{~m}^{2}$, the grids have 16 cluster head in both scenarios. The first scenario which considers static deployment based on the specified distance between $\mathrm{CH}$, and the second scenario that focused on random clustering is based on random deployment of the $\mathrm{CH}$. The time duration of the simulation is 120 seconds, maximum packet size to be sent is 512 bytes using SEP-E Routing protocol, Data Rate is 11 Mbps, Initial energy is $4 \mathrm{~mJ}$, Nodes deployment is random, Position Dynamic Channel type is Wireless Communication Bi directional. The simulation was implemented using MATLAB.

\subsection{Performance metrics}

There are many performance metrics applied for measuring the network quality of service, they are based on the network design and environment [23]. In this paper, a focusing on the performance metrics using to evaluate the residual and consumption of the energy during transmission, network lifetime, 
and throughput. These metrics include; consumed energy, residual energy, throughput which can be referred to the no. of the packets has been received at the BS during the rounds.

\subsubsection{Energy consumption}

The energy consumed by the transmitting nodes; it may be determined by measuring distances from the receiver. The transmitted energy is calculated for the transmission of $p$ bits of data over a distance $\mathrm{x}$ as [11]:

$$
\begin{aligned}
& E_{T}(p, x)=\left(E_{e l} * p+p * \epsilon_{f s} * x^{2}\right) \text { for } x<x_{0} \\
& E_{T}(p, x)=\left(E_{e l} * p+p * \epsilon_{m p} * x^{4}\right) \text { for } x>x_{0}
\end{aligned}
$$

where; $E_{e l}$ refers to electronic energy specified by issues such as digital coding or modulation.

$\epsilon_{f S}$ denotes to the amplified energy at free space model.

$\epsilon_{m p}$ is the amplified energy used in multi-path fading model.

$x_{0}$ is the threshold distance that is stated by:

$$
x_{0}=\sqrt{\epsilon_{f s} / \epsilon_{m p}}
$$

Energy consuming though receiving $p$ data bits is:

$$
E_{R}(P)=\left(E_{e l} \times P\right)
$$

Energy consuming by nodes in aggregate excessive $m$ messages of $\mathrm{p}$ bits each, which is estimated by:

$$
E_{D A}(m, p)=\left(E_{a g g} \times p \times m\right)
$$

where $E_{a g g}$ is the consuming energy per bit to aggregate of the message.

\subsubsection{Rounding time}

The total time for a complete round is calculated at BS. The BS also recognizes the cluster with the highest node number, and then computes the nodes number in it. When $u$ is the nodes number in the cluster, then a desired total time for one round is specified by:

$$
T=(u-1) t_{C M-C H}+(n-1) t_{C H-C H}+\frac{x}{v}+i \times t_{M}
$$

where; $t_{C M-C H}$ is the slot time, given to the node for sending data to $\mathrm{CH}$. Its the slot time assigned to a $\mathrm{CH}$ for sending data to its upper level $\mathrm{CH}$.

$n$ is the levels number.

$v$ is the data mule velocity.

$\mathrm{x}$ is the horizontal width of the network.

$i$ is the vertical lanes number, which is a number of APs that data mule halts collect the data.

$t_{M}$ is the time it takes with a data mule at each AP for collecting data from the $\mathrm{CH}$.

\subsubsection{Packet delivery ratio (PDR)}

PDR represents the packet ratio, which is sent by the application layer through simulation is being performed. It can be calculated theoretically by (7)

$$
(P D R) \%=\frac{\sum \text { packet Received by destination }}{\sum \text { packet sent by Source }} \times 100
$$

\subsubsection{Throughput}

It is an important parameter that is used in evaluating the packet numbers with successfully transferred between the source and destination. It can be resulting from dividing the communication channel to simulation time. Several factors influence the success of throughput at any network, like propagation losses, traffic and network congestion. Throughput has a negative effect with these factors. It may be represented and calculated theoretically by (8):

$$
\overline{T h r}=\frac{8 \times N_{r x}}{T_{\text {Sim }}}
$$

Design and implementation of grid based clustering in WSN using dynamic sink node (Aya Ahkam Kamil) 
where $; \overline{T h r}$ : the average throughput (bps).

$N_{r x}$ : the successfully received packet number (byte).

$T_{\text {Sim }}$ : the simulation time (second).

e. Network Lifetime

It is the minimum time duration ( $\mathrm{sec}$ ) of network operation in which the number of sensor nodes is dying or shut down at long simulations run performed.

\section{PROPOSED APPROACHES}

From the simulation, the detailed results, including design, analysis, and the specifications for achieving appropriate WSN performances will be shown. The aim is to keep the energy balancing among the cluster heads with the purpose of prolonging the network lifetime, by comparing the two scenarios, the first one is grid-based static sink node position, while the second scenario is grid-based dynamic sink node position network design. The performance of the two scenarios will be validated and compared via simulation using Matlab, and all simulation parameters for both scenarios are described above.

\subsection{Grid based clustering under static sink node position}

Although the design of the grid based-clustering in WSN improved the performance of the network, several observations can be made based on the following Figure 3, the number of clusters increases and distributed in equal distance in every grid of the network depending on the highest energy node to become the head in grids. But, the value of energy consumption of the nodes increases, because the sink node located in static position since the hole network nodes with similar initial energy, so the nodes closest to the sink node are faster exhausted energy compared to the far nodes from the sink. This result could be described with a fact that sensor nodes will be closest position for the $\mathrm{CH}$ at grid-based clustering, and the 16-cluster head deployment created on the specified distance among them. These nodes consume more power by sending the its packets and pass the packets from the farthest nodes to the sink nodes.

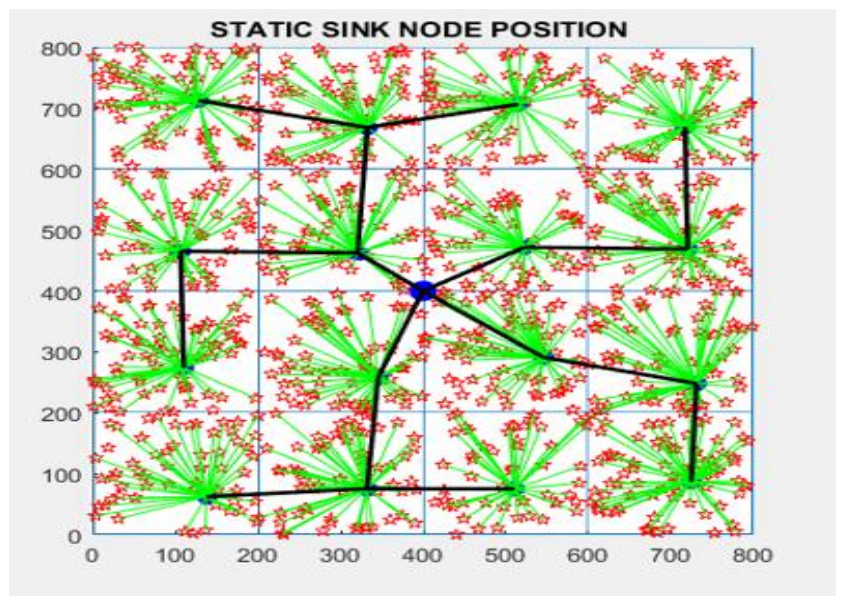

Figure 3. Grid based cluster head under static sink node position

\subsection{Grid based clustering under dynamic sink node position}

After implementing the scenario of static sink node position in grid-based clustering network for collecting data in WSN by utilizing multi hop forwarding, thus more energy is consumed close to the node round base station and rotating data to other one [24]. So, to solve the problem of energy consumption dynamic sink node position utilized for collecting data from sensors node and then stored it at base station during one round as shown in Figure 4(a, b, c, d). Every new round in the network the sink node has new position for balancing the energy consumption among multiple nodes entire network [25]. Comparative study of the two scenarios (static, dynamic) had been implemented with different metrics been simulated using MATLAB, depending on the static sink node and dynamic sink node results, a decision in which technique can be considered effective for collecting data from the sensor nodes. 


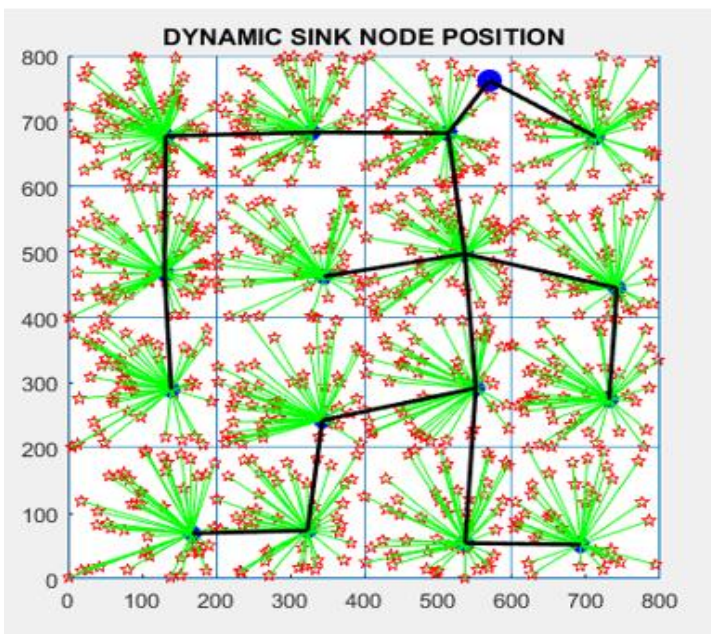

(a)

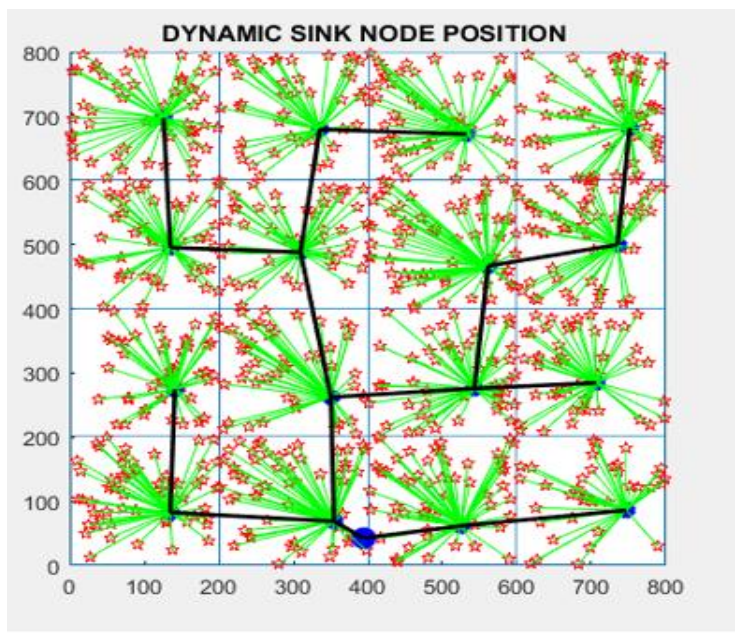

(c)

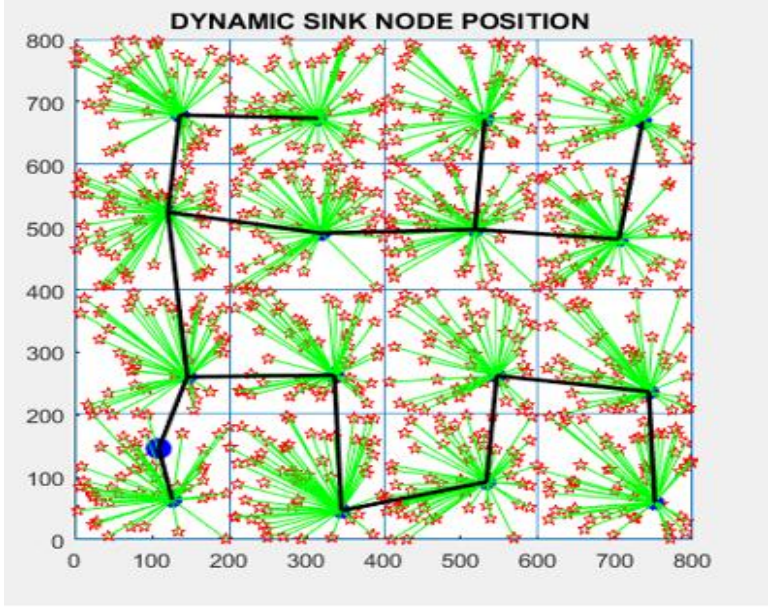

(b)

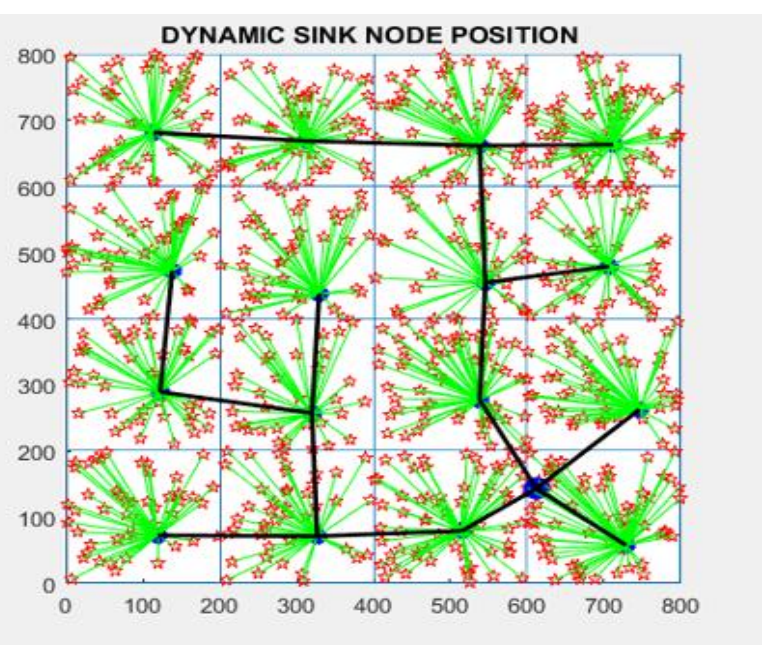

(d)

Figure 4. Dynamic sink node position in grid based cluster head network

\section{RESULT AND DISCUSSION}

Various performance metrics have been analyzed such as consumed and residual energy, in addition to the packets number sent to the base station, and the lifetime.

\subsection{Comsumed and residual energy}

\subsubsection{Grid based clustering energy under static sink node position}

The consumed and residual energy for the first scenario under static sink node position in WSN can observe in Figure 5 which shows the value of energy represented by residual and consumed. So, with this scenario's design, a consumed energy is high at the process compared to residual, this is due to the nodes that are closest to the sink are overburdened by an enormous traffic load besides the grids have furthest nodes trying to make a communication with neighboring clusters as the whole data region will be transmitting through them for connecting and then sending their packets to the sink node.

\subsubsection{Grid based clustering energy under dynamic sink node position the word "data" is plural, not singular}

The grid-based clustering with the most significant parameter evaluated is the consumed and residual energy at the end of each simulation using the dynamic position of the sink node and can be shown in Figure 6. With respect to this scenario, a decreasing in the energy consumption which causes a maximization of the residual energy so that an enhancement in the network performance. The energy

Design and implementation of grid based clustering in WSN using dynamic sink node (Aya Ahkam Kamil) 
consumed by sensor nodes per cluster decreases significantly as the position of the sink node had been changed in every round which solving the energy balancing problem. In contrast, static sink position does not guarantee such issues because in some cases data flow will go through a long path to reach the sink node consuming more energy with high traffic load around the sink node.

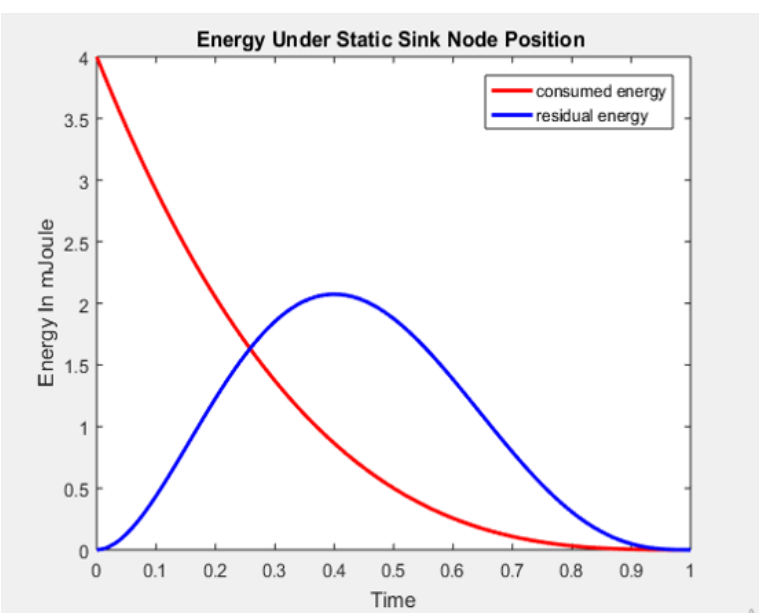

Figure 5. Residual and consumed energy for grid based clustering under static

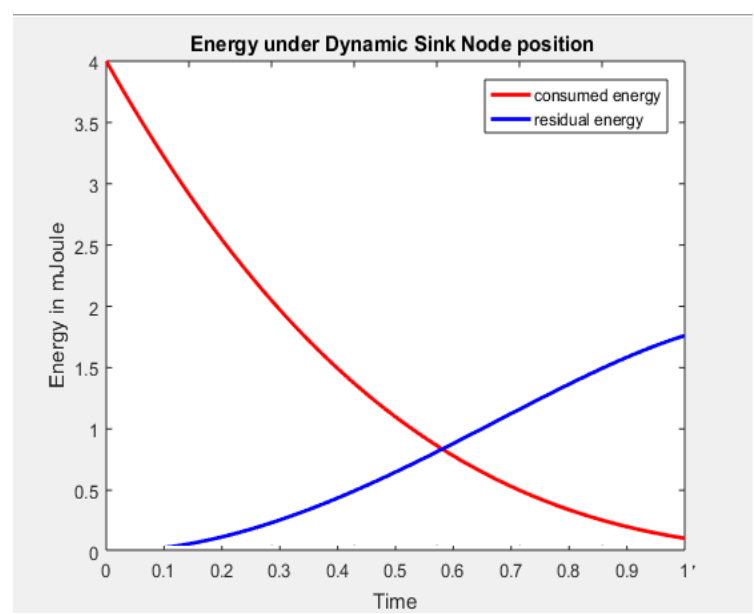

Figure 6. Residual and consumed energy for grid based clustering under dynamic

It can be noted from the comparisons between the static and dynamic sink node position that the residual energy increased, and energy consumption reach to the minimum value when positioned the sink node in multiple locations in the network, but the consumed energy increased when used the static position for the sink node in the wireless sensor network as shown in Figure 7. As shown in Figure 7, the relationship between the number of clusters (16 cluster head) in grid-based in WSN of 800 nodes and energy consumption for cluster-head based on the scenarios of static and dynamic position of the sink.

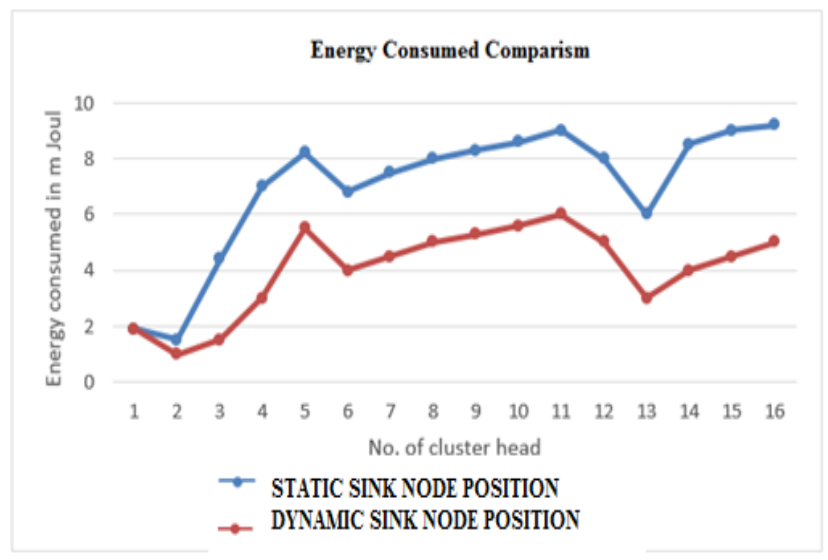

Figure 7. Energy consumed for grid-based clustering in WSN

\subsection{Troughput}

The performance of the two-design depending on the packet number being sent in one round can be analyzed with corresponding to the values of a throughput can be shown in Figure 8. A throughput comparison value between grid-based clustering under static and dynamic position of the sink node are shown. After round number 11 (11 iteration) the grid based static position will drop down, but the value of grid based dynamic position will increase continuously, in this round can compare the value of both scenarios as shown in Figure 8. 


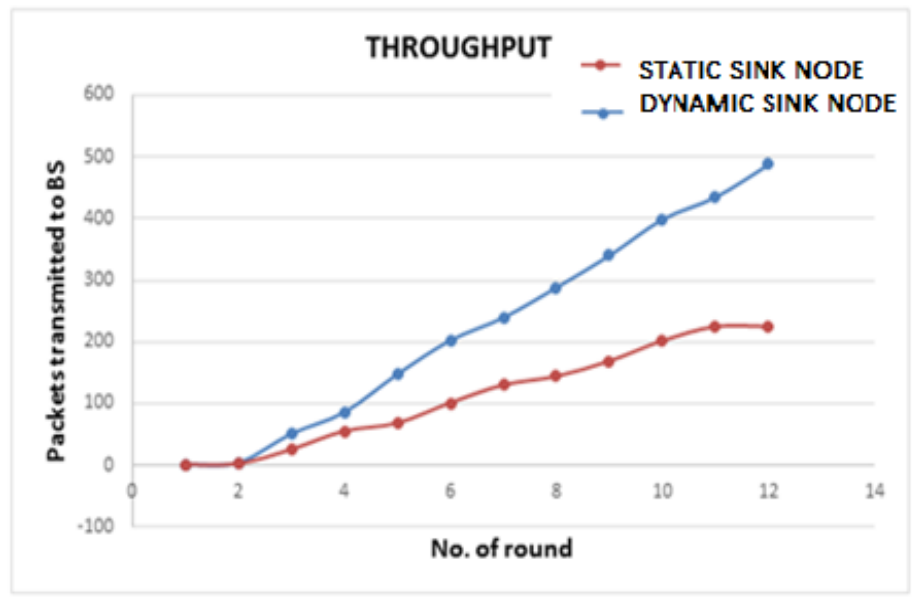

Figure 8. Throughput comparison between static and dynamic sink node position

\subsection{Network lifetime}

The procedure of the network lifetime with changed range of transmission can be seen in Figure 9. If the rounds number increased at a growing of network transmission, then a dynamic sink node position lifetime in grid based is extended more than that in static position.

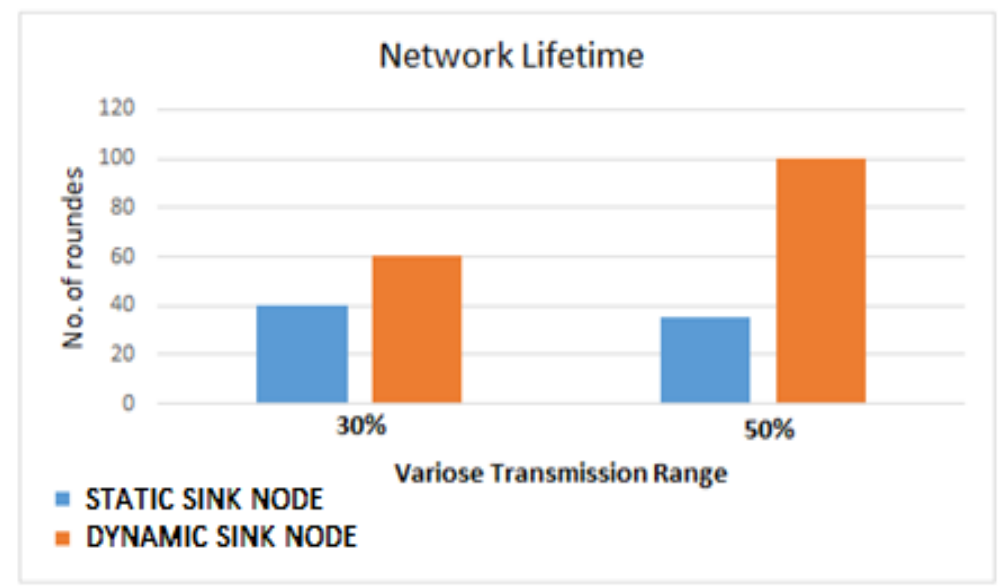

Figure 9. Network lifetime simulation at various transmission range

\section{CONCLUSION}

Grid based clustering is simpler and more feasible, and has so much advantage with respect to other method. In this paper, a proposed grid-based clustering under sink node mobility for balancing the traffic load, and minimizing energy consumption among all the nodes is tested. The routing protocols of multi-hop is suffering problems such as hot spot because of a huge traffic load nearby BSs. By applying static and dynamic algorithms, the transmission of the data are through vertically rectangular, as parallel lanes and hence a reduction in the hot spot problem occurs for balancing energy consumption with different points.

However, Grid based clustering head obtained to enhance results in terms of energy, with dynamic sink node position for balancing traffic load entire network, its used in long transmission sensor network, arrangement of packets scheduling doesn't take into account yet to reach an optimal solution in terms of synchronization of the packets. although routing protocol is taking into consideration, a trade-off will be produced between the transmitted range and communication distance. The optimal route can be created by all components of sensors which must consider and produce negligible energy procedure for transmission data in the future. 


\section{REFERENCES}

[1] S. Alam, D. De and A. Ray, "Analysis of Energy Consumption for IARP, RIP and STAR Routing Protocols in Wireless Sensor Networks," 2015 Second International Conference on Advances in Computing and Communication Engineering, Dehradun, pp. 11-16, 2015.

[2] M. M. Kareem, M. Ismail, M. A. Altahrawi, N. Arsad, M. F. Mansor and Adnan H. Ali, "Grid Based Clustering Technique in Wireless Sensor Network using Hierarchical Routing Protocol," 2018 IEEE 4th International Symposium on Telecommunication Technologies (ISTT), Selangor, Malaysia, pp. 1-5, 2018.

[3] Alaa Farhood, Maham Kamil Naji, Suhad Hasan Rhaif Hasan Rhaif, Adnan H. Ali, "Design and analysis of dual band integrated hexagonal shaped microstrip UWB antenna," Indonesian Journal of Electrical Engineering and Computer Science (IJEECS), vol. 15, no. 1, pp. 294-299, July 2019.

[4] M. A. Saad, Mustafa S. T., M. H. Ali, M. M. Hashim, M. Bin Ismail, and Adnan H. Ali, "Spectrum sensing and energy detection in cognitive networks," Indonesian Journal of Electrical Engineering and Computer Science, vol. 17 , no. 1 , pp. 464-471, 2020.

[5] H. M. Noman, Ali A. Abdulrazzaq, Marwah M. Kareem, and Adnan H. Ali, "Improvement investigation of the TCP algorithms with avoiding network congestion based on OPNET," 2nd International Conference on Sustainable Engineering Techniques, vol. 518, no. 5, 2019.

[6] K. M. Amrutha, P. Ashwini, D. K. Raj, G. K. Rani, and M. R. Mundada, "Energy efficient clustering and gridbased routing in wireless sensor networks," Proceedings of international conference on advances in computing, Springer, New Delhi, India, vol. 174, pp. 69-74, 2013.

[7] Pratibha Kevre, Laxmi Shrivastava, "Compare three reactive routing protocols in grid based cluster wireless sensor network using qualnet simulator," Journal of Communication Engineering \& Systems, vol 4, no. 1, pp. 6-12, 2014.

[8] C. X. Chen, Z. W. He, J. M. Kuang, and H. M. Sun, "Grid-based cluster head selection algorithm in wireless sensor networks," Applied Mechanics and Materials, vol. 427-429, pp. 1497-1501, 2013.

[9] Ahmed J. Abid, Adnan Hussein Ali, "Smart monitoring of the consumption of home electrical energy," International Journal of Emerging Trends \& Technology in Computer Science, vol. 47, no. 2, pp. 142-148, 2017.

[10] S. Pradhan and K. Sharma, "Cluster head rotation in wireless sensor network: A simplified approach," International Journal of Sensor and Its Applications for Control Systems, vol. 4, no. 1, pp. 1-10, 2016.

[11] A. Pampatwar and Asha S., "Routing protocol for WSN based on an energy efficient even grid clustering," International Journal of Innovative Research in Computer and Communication Engineering, vol. 5, no. 4, pp. 321-326, 2017.

[12] K. Lalitha, R. Thangarajan, S. K. Udgata, C. Poongodi, and A. P. Sahu, "GCCR: An efficient grid based clustering and combinational routing in wireless sensor networks," Wireless Personal Communications, vol. 97, no. 1, pp. 1075-1095, 2017.

[13] H. Farman, H. Javed, J. Ahmad, B. Jan, and M. Zeeshan, "Grid-based hybrid network deployment approach for energy efficient wireless sensor networks," Journal of Sensors, vol. 2016, pp. 1-14, 2016.

[14] N. Ismat, R. Qureshi, and M. U. Imam, "Energy and round time estimation method for mobile wireless sensor networks," Mehran University Research Journal of Engineering \& Technology, vol. 37, no. 1, pp. 105-118, 2018.

[15] Z. Chen and H. Shen, "A grid-based reliable multi-hop routing protocol for energy-efficient wireless sensor networks," International Journal of Distributed Sensor Networks, vol. 14, no. 3, 2018.

[16] A. H. Ali and A. D. Farhood, 'Design and Performance analysis of the WDM schemes for radio over fiber system with different fiber propagation losses," Fibers, vol. 7, no. 19, 2019.

[17] Ahmed J. Abid, Fawzi M. Al-Naima, and Adnan H. Ali, "Comprehensive modeling of PV array based on proteus software," International Journal of Applied Engineering Research, vol. 13, no. 6, pp. 4440-4447, 2018.

[18] A. Maroosi and R. C. Muniyandi, "Accelerated simulation of membrane computing to solve the n-queens problem on multi-core," International Conference on Swarm, Evolutionary, and Memetic Computing, Springer, Cham, pp. 257-267, 2013.

[19] M. K. Naji, A. D. Farhood, A. H. Ali, "Novel design and analysis of RF MEMS shunt capacitive switch for radar and satellite communications," Indonesian Journal of Electrical Engineering and Computer Science (IJEECS), vol. 15, no. 2, pp. 971-978, 2019.

[20] S. Din, A. Paul, A. Ahmad, and J. H. Kim, "Energy efficient topology management scheme based on clustering technique for software defined wireless sensor network," Peer-to Peer Networking and Applications, vol. 12, no. 12 , pp. 348-356, 2019.

[21] Adnan H. Ali, Hayder J. Alhamdane, Begared S. Hassen, "Design analysis and performance evaluation of the WDM integration with CO-OFDM system for radio over fiber system," Indonesian Journal of Electrical Engineering and Computer Science (IJEECS), vol. 15, no. 2, pp. 870-878, 2019.

[22] Anas A. Hussien, Adnan H. Ali, "Comprehensive investigation of coherent optical OFDM-RoF employing 16QAM external modulation for long-haul optical communication system," International Journal of Electrical and Computer Engineering, vol. 10, no. 3, pp. 2607-2616, June 2020

[23] A. Sarkar and T. S. Murugan, "Cluster head selection for energy efficient and delay-less routing in wireless sensor network," Wireless Networks, vol. 25, no. 1, pp. 303-320, 2019.

[24] Samir A. Lafta, Aktham H. Ali, Marwah M. Kareem, Yasser A. Hussein, and Adnan H. Ali, "Performance simulation of broadband multimedia wireless networks simulation based on OPNET," Indonesian Journal of Electrical Engineering and Computer Science, vol. 17, no. 1, pp. 1-9, 2020.

[25] Adnan H. Ali, "Performance evaluation of Wi-Fi physical layer based QoS systems on fiber using OPNET modeler," International Journal of Soft Computing and Engineering, vol. 5, no. 3, pp. 79-86, July 2015. 